\title{
Sulfated tungstate catalyzed hydration of alkynes
}

\author{
Sachin D. Veer, Sagar P. Pathare, and Krishnacharya G. Akamanchi* \\ Department of Pharmaceutical Sciences and Technology, Institute of Chemical Technology, \\ Matunga, Mumbai 400 019, India \\ E-mail:kgap@rediffmail.com
}

DOI: http://dx.doi.org/10.3998/ark.5550190.p009.493

\begin{abstract}
A heterogeneous catalyst based on sulfated tungstate has been developed for the environmentally benign hydration of alkynes to the corresponding ketones in high yields (84-95\%). Attractive features of the protocol are its generality, as evidenced by its successful application to alkyl and aryl alkynes with variable functionalities, mild and solvent free conditions, easy product isolation and reusability of the catalyst.
\end{abstract}

Keywords: Alkynes, hydration, sulfated tungstate, heterogeneous catalysts, ketones

\section{Introduction}

The catalytic hydration of alkynes, leading to the formation of aldehydes and ketones is of considerable interest because of availability of alkynyl substrates and the fundamental importance of aldehydes and ketones for bulk and fine chemical industries. Alkyne hydration has been employed in the synthesis of active pharmaceutical ingredients, such as azaperone, benperidol, droperidol and fexofenadine. The hydration of terminal alkynes can give either a methyl ketone (Markovnikov addition) or an aldehyde (anti-Markovnikov addition), however, in most of the cases Markovnikov addition occurs giving methyl ketones exclusively. Classical approaches, towards hydration of alkynes, use $\mathrm{HgO}-\mathrm{H}_{2} \mathrm{SO}_{4}{ }^{1,2}$ and $\mathrm{HgO}-\mathrm{BF}_{3}{ }^{3,4}$ as catalysts and need strong acidic conditions but mercury toxicity and the requirement of strong acids are major drawbacks of these approaches. Brønsted acids, ${ }^{5}$ various transition metals including $\mathrm{Pd},{ }^{6} \mathrm{Ir}^{7} \mathrm{Pt}^{8}$ and $\mathrm{Fe},{ }^{9}$ have been examined as catalysts and were viable for the hydration. Gold complexes ${ }^{10,11}$ have also been employed and require, in many cases, silver salts ${ }^{12}$ as a co-catalyst to enhance the catalytic activity. Recently, $\mathrm{AgSbF}_{6}$ catalyzed hydration of alkynes has been reported. ${ }^{13}$ All these systems have drawbacks, predominantly high cost of the metals, recovery and reuse of catalysts, and use of strong acids as co-catalysts, which may cause chemoselectivity and substrate compatibility problems.

Heterogeneous catalysts have received attention as green catalysts because of their inherent advantageous ease of recovery and potential for recyclability. Kobayashi and co-workers have 
reported hydration of 4-ethynyltoluene over alkylated polystyrene supported sulfonic acid using a catalytic amount of Brønsted acid. ${ }^{14}$ However, the catalytic activity was low and method is limited to 4-ethynyltoluene only. Recently, AuI containing mesoporous silica ${ }^{15}$ catalyzed hydrations of alkynes, including aromatic and aliphatic alkynes, have been disclosed. Although, this catalyst showed high efficiency, it still requires $\mathrm{H}_{2} \mathrm{SO}_{4}$ as co-catalyst. With these limited methods, and many drawbacks, there is scope for newer, especially greener, methods.

Recently, we introduced sulfated tungstate as a mild solid acid and placed it above silica in acidity and demonstrated its effectiveness as a green catalyst for many reactions including amidation, ${ }^{16}$ transamidation, ${ }^{17}$ Ritter reaction, ${ }^{18} \mathrm{~N}$-formylation, ${ }^{19}$ Biginelli, ${ }^{20}$ Kindler, ${ }^{21}$ Willgerodt-Kindler, ${ }^{22}$ Strecker reactions ${ }^{23}$ and $\mathrm{N}$-alkylations. ${ }^{24}$ Attributes of sulfated tungstate are its ease of preparation, mild acidity, stability, and reusability. These attributes encouraged us to further explore and expand its applications to firmly establish its versatility. Herein, investigations related to its potential as a catalyst for the hydration of alkynes to form methyl ketones are disclosed.

\section{Result and Discussion}

Preliminary investigations to study the suitability of sulfated tungstate as a catalyst for hydration of alkynes and optimization of reaction conditions involved the use of phenylacetylene as model substrate (Scheme 1).

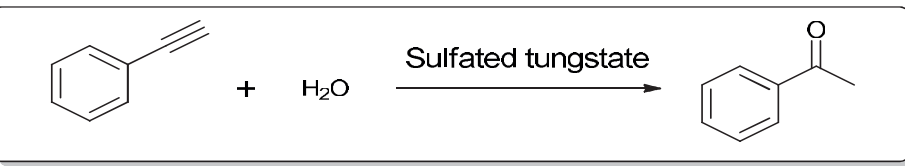

Scheme 1. Hydration of phenyl alkyne using sulfated tungstate.

Investigations were carried out to establish the catalytic role of sulfated tungstate and to optimize the reaction conditions (Table 1). Reactions were carried out under solvent free conditions, both in the presence and absence of sulfated tungstate. In the presence of the catalyst $\left(20 \mathrm{wt} \%\right.$ ) at $100{ }^{\circ} \mathrm{C}$ after $3 \mathrm{~h}$ phenylacetylene was converted to acetophenone in $95 \%$ yield, but in the absence of catalyst the reaction did not proceed in $12 \mathrm{~h}$ and the substrate remained unreacted, thus establishing the catalytic role (Table 1, entries 1 and 5). Further experiments to optimize the quantity of sulfated tungstate needed to find that $20 \mathrm{wt} \%$ was sufficient to produce in $3 \mathrm{~h}$ the product in an isolated yield of $95 \%$ (Table 1, entries 2-7). When higher percentages were used stirring the reaction mixture became increasingly difficult and at $100 \%$ almost all substrate adsorbed on the catalyst leading to erratic results. Experiments were also carried out at room temperature, 60 and $80{ }^{\circ} \mathrm{C}$, using $20 \mathrm{wt} \%$ of the catalyst. The reaction failed to occur at room temperature (Table 1 entry 6 ) and yields declined to 41 and $66 \%$ at 60 and $80{ }^{\circ} \mathrm{C}$, respectively 
(Table 1, entries 7 and 8). Since water is a reactant, an experiment was carried out in the presence of excess of water and the yield remained unaffected indicating that the water quantity used in the initial experiments was adequate. As regards the use of solvent, reactions were carried out in ethanol, ethyl acetate and toluene. All the solvents were suitable but gave relatively lower yields, which could be attributed to lower reaction temperatures in case of ethanol and ethyl acetate (Table 1, entries 9 and 12) and the limited water solubility in toluene (Table 1, entry 15). Experiments with higher loading of catalyst were possible in solvents and the results with 50 and $100 \mathrm{wt} \%$ are included in Table 1. In all cases with higher catalyst loading yields were lower with substrate remaining unreacted (Table 1 , entries 9 to 17).

Table 1. Results of optimisation studies ${ }^{a}$

\begin{tabular}{cccccc}
\hline Entry & Solvent & $\begin{array}{c}\text { Sulfated } \\
\text { tungstate }(\mathrm{wt} \%)\end{array}$ & Temp $\left({ }^{\circ} \mathrm{C}\right)$ & Time $(\mathrm{min})$ & Yield $^{b}(\%)$ \\
\hline 1 & Neat & Nil & 100 & 12 & Nil \\
2 & Neat & 1 & 100 & 6 & 22 \\
3 & Neat & 5 & 100 & 6 & 56 \\
4 & Neat & 10 & 100 & 6 & 74 \\
$\mathbf{5}^{c}$ & Neat & $\mathbf{2 0}$ & $\mathbf{1 0 0}$ & $\mathbf{3}$ & $\mathbf{9 5}$ \\
6 & Neat & 20 & $\mathrm{rt}$ & 12 & Nil \\
7 & Neat & 20 & 60 & 12 & 41 \\
8 & Neat & 20 & 80 & 12 & 66 \\
9 & Ethanol & 20 & Reflux & 6 & 78 \\
10 & Ethanol & 50 & Reflux & 6 & 75 \\
11 & Ethanol & 100 & Reflux & 6 & 50 \\
12 & Ethyl acetate & 20 & Reflux & 6 & 64 \\
13 & Ethyl acetate & 50 & Reflux & 6 & 60 \\
14 & Ethyl acetate & 100 & Reflux & 6 & 50 \\
15 & Toluene & 20 & Reflux & 6 & 35 \\
16 & Toluene & 50 & Reflux & 6 & 30 \\
17 & Toluene & 100 & Reflux & 6 & 20 \\
\hline
\end{tabular}

${ }^{a}$ Reaction condition: phenylacetylene $(1.00 \mathrm{~g}, 9.79 \mathrm{mmol})$ and water $(0.74 \mathrm{~g}, 39.16 \mathrm{mmol})$ at different temperature. ${ }^{b}$ Isolated yield. ${ }^{c}$ Bold indicates optimum conditions used in subsequent studies.

To establish the scope of the protocol, various aromatic and aliphatic alkynes were examined (Table 2). All the substrates were efficiently converted into the corresponding methyl ketones in moderate to high yields. Aromatic substrates carrying various electron donating or withdrawing groups at para or meta positions underwent reaction smoothly to the corresponding carbonyl compounds in moderate to high yields, indicating that substituents have no influence on the 
reaction outcome (Table 2, entries 2-9). Aliphatic terminal alkynes such as 4-pentyn-1-ol and prop2-ynyl benzoate can also be hydrated but these reactions were slow and required longer reaction times to achieve adequate yields of the expected products (Table 2, entries 10 and 11). A reaction was attempted with diphenylacetylene, a substrate with an internal alkyne, but it was unreactive (Table 2, entry 12). The failure of diphenylacetylene (internal alkyne) to react was tentatively attributed to its non polar nature and higher stability owing to extended conjugation.

The work-up procedure was simple; sulfated tungstate was filtered off after diluting the reaction mixture with ethyl acetate, followed by concentration and purification by chromatography.

Table 2. Hydration reactions of various alkynes in the presence of sulfated tungstate ${ }^{a}$

E


${ }^{a}$ Reaction conditions: Phenylacetylene (1 equiv.), water (4 equiv.) and sulfated tungstate (20 $\mathrm{wt} \%$ ) under solvent free at $100{ }^{\circ} \mathrm{C} .{ }^{b}$ Isolated yield. ${ }^{c}$ All products are known and were identified by their melting point (if any), IR and ${ }^{1} \mathrm{H}-\mathrm{NMR}$ spectra as per literature. ${ }^{d} \mathrm{NR}=$ No reaction.

A catalyst reusability study was performed, by recycling the catalyst after isolation and drying, without makeup of the handling losses which stood at $<5 \%$, and the catalyst was stable and reusable four times without any significant loss of activity with marginal drop in yield from 95\% with fresh catalyst to $91 \%$ after a fourth recycle.

To show that the reaction is heterogeneous, a standard metal leaching experiment was conducted by hot filtration method. Two sets of experiments were conducted and both reactions proceeded for $30 \mathrm{~min}$. One experiment was worked up, while from the other, the catalyst was filtered off and the reaction was continued at the same reaction temperature of $100{ }^{\circ} \mathrm{C}$ for $3 \mathrm{~h}$ and worked up. The yields obtained in both cases, 16 and 18\%, respectively, were practically the same, indicating that no homogeneous catalysis was involved.

\section{Conclusions}

In conclusion, sulfated tungstate has been established as a highly efficient mildly acidic heterogeneous catalyst for the hydration of terminal alkynes. This new protocol has advantages of catalyst reusability, solvent-free conditions, and easy workup procedures, thus scoring high on eco-friendliness.

\section{Experimental Section}

Reagents. All chemicals were purchased from Spectrochem Pvt. Ltd. India and were used without further purification.

\section{Preparation of sulfated tungstate ${ }^{16}$}

Anhydrous sodium tungstate $(32.9 \mathrm{~g}, 0.1 \mathrm{~mol})$ was added portion wise, maintaining the temperature between 0 to $5{ }^{\circ} \mathrm{C}$, to a stirred solution of chlorosulfonic acid ( $\left.23.2 \mathrm{~g}, 0.2 \mathrm{~mol}\right)$ in chloroform $(150 \mathrm{~mL})$ contained in a $250 \mathrm{~mL}$ round bottom flask fitted with $\mathrm{CaCl}_{2}$ drying tube, placed in an ice bath. After completion of addition, the mixture was stirred further for $1 \mathrm{~h}$. The pale yellow solid obtained was filtered, washed repeatedly with deionized water until filtrate was neutral and free from chloride ions (detected by $\mathrm{AgNO}_{3}$ test) and dried in an oven for $2 \mathrm{~h}$ at 100 ${ }^{\circ} \mathrm{C}$ to get sulfated tungstate $(33 \mathrm{~g}, 86 \% \mathrm{w} / \mathrm{w})$.

General procedure for hydration of phenylacetylene (Table 2, entry 1). Sulfated tungstate $(0.20 \mathrm{~g}, 20 \mathrm{wt} \%)$ was added to a mixture of phenylacetylene $(1.00 \mathrm{~g}, 9.79 \mathrm{mmol})$ and water $(0.74$ $\mathrm{g}, 39.16 \mathrm{mmol}$ ) and the resulting suspension was stirred at $100{ }^{\circ} \mathrm{C}$. The progress of the reaction was monitored by TLC. After the completion of the reaction, the reaction mixture was cooled, 
diluted with EtOAc $(10 \mathrm{~mL})$ and filtered. The catalyst was washed with EtOAc $(10 \mathrm{~mL})$, combined organic layer was concentrated under reduced pressure to give crude product which was purified by chromatography on silica gel (\#60-120) with $n$-hexane/EtOAc $(90: 10)$ as eluent to get pure $(1.12 \mathrm{~g})$ acetophenone as a colorless liquid.

Acetophenone (Table 2, entry 1). Colorless liquid; $R_{\mathrm{f}} 0.47$ ( $n$-hexane/EtOAc, 90:10); IR: 1681, $1598,1582,1448,1358,1263,1180,1078,1024 \mathrm{~cm}^{-1} ;{ }^{1} \mathrm{H} \mathrm{NMR}\left(300 \mathrm{MHz} ; \mathrm{CDCl}_{3} ; \mathrm{Me}_{4} \mathrm{Si}\right): \delta_{\mathrm{H}}$ 7.93-7.95 (m, 2H), 7.52-7.56 (m, 1H), 7.44-7.46 (m, 2H), $2.58(\mathrm{~s}, 3 \mathrm{H})$.

4-Methylacetophenone (Table 2, entry 2). Colorless liquid; $R_{\mathrm{f}} 0.47$ ( $n$-hexane/EtOAc, 90:10); IR: $1687,1599,1586,1450,1354,1265,1181,1077,1026 \mathrm{~cm}^{-1} ;{ }^{1} \mathrm{H}$ NMR (300 MHz; $\mathrm{CDCl}_{3}$; $\left.\mathrm{Me}_{4} \mathrm{Si}\right): \delta_{\mathrm{H}} 7.83(\mathrm{~d}, J 8.1 \mathrm{~Hz}, 2 \mathrm{H}), 7.23(\mathrm{~d}, J 8.1 \mathrm{~Hz}, 2 \mathrm{H}), 2.38(\mathrm{~s}, 3 \mathrm{H}), 2.55(\mathrm{~s}, 3 \mathrm{H})$.

4-Aminoacetophenone (Table 2, entry 3). Brown solid, mp $102-104{ }^{\circ} \mathrm{C}$ (lit. ${ }^{26} 104-106{ }^{\circ} \mathrm{C} ; R_{\mathrm{f}}$ 0.13 (n-hexane/EtOAc, 90:10); IR (KBr): 3333, 2924, $1642 \mathrm{~cm}^{-1} ;{ }^{1} \mathrm{H}$ NMR (300 MHz; $\mathrm{CDCl}_{3}$; $\mathrm{Me}_{4} \mathrm{Si}$ ): $\delta_{\mathrm{H}} 7.79(\mathrm{~d}, J 8.6 \mathrm{~Hz}, 2 \mathrm{H}), 6.63(\mathrm{~d}, J 8.6 \mathrm{~Hz}, 2 \mathrm{H}), 4.11$ (br s, 2H), $2.48(\mathrm{~s}, 3 \mathrm{H})$.

4-Hydroxyacetophenone (Table 2, entry 4): colorless solid, mp $110-111^{\circ} \mathrm{C}$ (lit., ${ }^{29} 109-111{ }^{\circ} \mathrm{C}$ ); $R_{\mathrm{f}} 0.13$ ( $n$-hexane/EtOAc, 90:10); IR (KBr): 3311, 1663, 1614, 1507, 1239, $1072 \mathrm{~cm}^{-1} ;{ }^{1} \mathrm{H}$ NMR $\left(300 \mathrm{MHz} ; \mathrm{CDCl}_{3} ; \mathrm{Me}_{4} \mathrm{Si}\right): \delta_{\mathrm{H}} 7.59(\mathrm{~s}, 1 \mathrm{H}), 7.89(\mathrm{~d}, J 8.5 \mathrm{~Hz}, 2 \mathrm{H}), 6.92(\mathrm{~d}, J 8.5 \mathrm{~Hz}, 2 \mathrm{H}), 2.56$ (s, $3 \mathrm{H})$.

4-Methoxyacetophenone (Table 2, entry 5). Colorless solid; mp $36-38{ }^{\circ} \mathrm{C}$ (lit., ${ }^{13} 37-38{ }^{\circ} \mathrm{C}$ ); $R_{\mathrm{f}}$ 0.31 (n-hexane/EtOAc, 90:10); IR (KBr): 1683, 1597, 1584, $1450 \mathrm{~cm}^{-1} ;{ }^{1} \mathrm{H}$ NMR (300 MHz; $\left.\mathrm{CDCl}_{3} ; \mathrm{Me}_{4} \mathrm{Si}\right): \delta_{\mathrm{H}} 7.89(\mathrm{~d}, J 9.0 \mathrm{~Hz}, 2 \mathrm{H}), 6.88(\mathrm{~d}, J 9.0 \mathrm{~Hz}, 2 \mathrm{H}), 3.81(\mathrm{~s}, 3 \mathrm{H}), 2.50(\mathrm{~s}, 3 \mathrm{H})$.

4-Chloroacetophenone (Table 2, entry 6). Colorless liquid; $R_{\mathrm{f}} 0.45$ ( $n$-hexane/EtOAc, 90:10); IR: 1696, 1590, 1588, $1454 \mathrm{~cm}^{-1} ;{ }^{1} \mathrm{H} \mathrm{NMR}\left(300 \mathrm{MHz} ; \mathrm{CDCl}_{3} ; \mathrm{Me}_{4} \mathrm{Si}\right): \delta_{\mathrm{H}} 7.86(\mathrm{~d}, J 8.3 \mathrm{~Hz}, 2 \mathrm{H})$, 7.39 (d, J $8.3 \mathrm{~Hz}, 2 \mathrm{H}), 2.55$ (s, 3H).

3-Chloroacetophenone (Table 2, entry 7). Colorless liquid; $R_{\mathrm{f}} 0.45$ ( $n$-hexane/EtOAc 90:10); IR: $1688,1572,1422,1358,1251 \mathrm{~cm}^{-1} ;{ }^{1} \mathrm{H}$ NMR $\left(300 \mathrm{MHz} ; \mathrm{CDCl}_{3} ; \mathrm{Me}_{4} \mathrm{Si}\right): \delta_{\mathrm{H}} 7.52(\mathrm{dd}, J 2.0,1.2$ $\mathrm{Hz}, 1 \mathrm{H}), 7.31-7.40$ (m, 2H), 7.27-7.29 (m, 1H), 2.62 (s, 3H).

4-Bromoacetophenone (Table 2, entry 8). Colorless liquid; $R_{\mathrm{f}} 0.43$ ( $n$-hexane/EtOAc, 90:10); IR: $1673,1587,1395,1269,1078 \mathrm{~cm}^{-1} ;{ }^{1} \mathrm{H}$ NMR $\left(300 \mathrm{MHz} ; \mathrm{CDCl}_{3} ; \mathrm{Me}_{4} \mathrm{Si}\right): \delta_{\mathrm{H}} 7.79(\mathrm{~d}, J 8.7 \mathrm{~Hz}$, 2H), $7.58(\mathrm{~d}, J 8.7 \mathrm{~Hz}, 2 \mathrm{H}), 2.56(\mathrm{~s}, 3 \mathrm{H})$.

3-Bromoacetophenone (Table 2, entry 9). Colorless liquid; $R_{\mathrm{f}} 0.43$ ( $n$-hexane/EtOAc, 90:10); IR: $1672,1586,1396,1270,1077 \mathrm{~cm}^{-1} ;{ }^{1} \mathrm{H}$ NMR $\left(300 \mathrm{MHz} ; \mathrm{CDCl}_{3} ; \mathrm{Me}_{4} \mathrm{Si}\right): \delta_{\mathrm{H}} 8.01-8.03(\mathrm{~m}$, $1 \mathrm{H}), 7.82-7.84(\mathrm{~m}, 1 \mathrm{H}), 7.63-7.65(\mathrm{~m}, 1 \mathrm{H}), 7.29-7.30(\mathrm{~m}, 1 \mathrm{H}), 2.54(\mathrm{~s}, 3 \mathrm{H})$.

5-Hydroxy-2-pentanone (Table 2, entry 10). Colorless liquid; $R_{\mathrm{f}} 0.18$ ( $n$-hexane/EtOAc, 90:10); IR: 3450, 1720, $1040 \mathrm{~cm}^{-1} ;{ }^{1} \mathrm{H}$ NMR $\left(300 \mathrm{MHz} ; \mathrm{CDCl}_{3} ; \mathrm{Me}_{4} \mathrm{Si}\right): \delta_{\mathrm{H}} 3.64$ (t, $\left.J 6.2 \mathrm{~Hz}, 2 \mathrm{H}\right), 2.30-$ $2.55(\mathrm{~m}, 4 \mathrm{H}), 1.66-2.00(\mathrm{~m}, 2 \mathrm{H}), 1.26-1.28(\mathrm{~m}, 1 \mathrm{H})$.

2-Oxopropyl benzoate (Table 2, entry 11). Colorless liquid; $R_{\mathrm{f}} 0.47$ ( $n$-hexane/EtOAc, 90:10); IR: $1726,1741 \mathrm{~cm}^{-1} ;{ }^{1} \mathrm{H}$ NMR $\left(300 \mathrm{MHz} ; \mathrm{CDCl}_{3} ; \mathrm{Me}_{4} \mathrm{Si}\right): \delta_{\mathrm{H}} 7.97-8.00(\mathrm{~m}, 2 \mathrm{H}), 7.45-7.50(\mathrm{~m}$, $1 \mathrm{H}), 7.32-7.36(\mathrm{~m}, 2 \mathrm{H}), 4.77(\mathrm{~s}, 2 \mathrm{H}), 2.10(\mathrm{~s}, 3 \mathrm{H})$. 


\section{Acknowledgements}

The authors thank the University Grant Commission of India for financial support.

\section{References}

1. Kutscheroff, J. M. Chem. Ber. 1884, 17, 13. http://dx.doi.org/10.1002/cber.18840170105

2. Kutscheroff, M. G. Chem. Ber. 1909, 42, 2759. http://dx.doi.org/ 10.1002/cber.190904202197

3. Hennion, G. F.; Killian, D. B.; Vaughn, T. H.; Nieuwland, J. A. J. Am. Chem. Soc. 1934, 56, 1130 .

http://dx.doi.org/10.1021/ja01320a039

4. Nieuwland, J. A.; Vogt, R. R.; Foohey, W. L. J. Am. Chem. Soc. 1930, 52, 1018. http://dx.doi.org/10.1021/ja01366a026

5. Tsuchimoto, T.; Joya, T.; Shirakawa, E.; Kawakami, Y. Synlett 2000, 1777. http://dx.doi.org/10.1055/s-2000-8707

6. Fukuda, Y.; Shiragami, H.; Uchimoto, K.; Nozaki, H. J. Org. Chem. 1991, 56, 5816. http://dx.doi.org/10.1021/jo00020a024

7. Kanemitsu, H.; Uehara, K.; Fukuzumi, S.; Ogo, S. J. Am. Chem. Soc. 2008, 130, 17141. http://dx.doi.org/ 10.1021/ja807254d

8. Francisco, L. W.; Moreno, D. A.; Atwood, J. D. Organometallics 2001, 20, 4237. http://dx.doi.org/10.1021/om0104870

9. Wu, X. F.; Bezier, D.; Darcel, C. Adv. Syn. Catal. 2009, 351, 367. http://dx.doi.org/ 10.1002/adsc.200800666

10. Mizushima, E.; Sato, K.; Hayashi, T.; Tanaka, M. Angew. Chem., Int. Ed. 2002, 41, 4563. http://dx.doi.org/10.1002/1521-3773(20021202)41:23<4563::AID-ANIE4563>3.0.CO;2-U

11. Casado, R.; Contel, M.; Laguna, M.; Romero, P.; Sanz, S. J. Am. Chem. Soc. 2003, 125, 11925. http://dx.doi.org/10.1021/ja036049x

12. Marion, N.; Ramon, R. S.; Nolan, S. P. J. Am. Chem. Soc. 2009, 131, 448. http://dx.doi.org/10.1021/ja809403e

13. Bui, M.; Thuong, T.; Mann, A.; Wagner, A. Chem. Comm. 2012, 48, 434. http://dx.doi.org/10.1039/C1CC12928G

14. Iimura, S.; Manabe, K.; Kobayashi, S. Org. Biomol. Chem. 2003, 1, 2416. http://dx.doi.org/10.1039/B305622H

15. Huang, J.; Zhu, F.; He, W.; Zhang, F.; Wang, W.; Li, H. J. Am. Chem. Soc. 2010, 132, 1492. http://dx.doi.org/10.1021/ja909596a 
16. Chaudhari, P. S.; Salim, S. D.; Sawant, R. V.; Akamanchi, K. G. Green Chem. 2010, 12, 1707.

http://dx.doi.org/10.1039/C0GC00053A

17. Katkar, K. V.; Chaudhari, P. S.; Akamanchi, K. G. Green Chem. 2011, 13, 835. http://dx.doi.org/10.1039/C0GC00759E

18. Pathare, S. P.; Akamanchi, K. G. RSC Adv. 2013, 3, 7697. http://dx.doi.org/10.1039/C3RA00127J

19. Pathare, S. P.; Akamanchi, K. G. Tetrahedron Lett. 2012, 53, 3259. http://dx.doi.org/10.1016/j.tetlet.2012.04.058

20. Salim, S. D.; Akamanchi, K. G. Catal. Comm. 2011, 12, 1153. http://dx.doi.org/10.1016/j.catcom.2011.02.018

21. Pathare, S. P.; Chaudhari, P. S.; Akamanchi, K. G. Appl. Catal. A: General 2012, 125, 425. http://dx.doi.org/10.1016/j.apcata.2012.03.012

22. Salim, S. D.; Pathare, S. P.; Akamanchi, K. G. Catal. Comm. 2011, 13, 78. http://dx.doi.org/10.1016/j.catcom.2011.06.022

23. Pathare, S. P.; Akamanchi, K. G. Tetrahedron Lett. 2012, 53, 871. http://dx.doi.org/10.1016/j.tetlet.2011.12.027

24. Pathare, S. P.; Akamanchi, K. G. Appl. Catal. A: General 2012, 452, 29. http://dx.doi.org/10.1016/j.apcata.2012.11.017

25. Kulkarni, M. G.; Bagale, S. M.; Shinde, M. P.; Gaikwad, D. D.; Borhade, A. S.; Dhondge, A. P.; Chavhan, S. W.; Shaikh, Y. B.; Ningdale, V. B.; Desai, M. P.; Birhade, D. R. Tetrahedron Lett. 2009, 50, 2893. http://dx.doi.org/10.1016/j.tetlet.2009.03.173

26. Chen, T.; Cai, C. Catal.Comm. 2015, 65, 102. http://dx.doi.org/10.1016/j.catcom.2015.03.003

27. Chen, Z-W.; Ye, D-N.; Qian, Y-P.; Ye, M.; Liu, L-X. Tetrahedron 2013, 69, 6116. http://dx.doi.org/10.1016/j.tet.2013.05.057

28. Mameda, N.; Peraka, S.; Marri, M. R.; Kodumuri, S.; Chevell, D.; Gutt, N.; Nama, N. Appl. Catal. A: General 2015, 505, 213. http://dx.doi.org/10.1016/j.apcata.2015.07.038

29. Gohain, M.; Plessis, M. du.; Tonder, J. H.; Bezuidenhoudt, B. C. B. Tetrahedron Lett. 2014, 55, 2082.

http://dx.doi.org/10.1016/j.tetlet.2014.02.048 